\title{
Mitteilungen der Gastroenterologischen Arbeitsgemeinschaft Rheinland-Pfalz/Saarland (GARPS)
}

Gastroenterologe 2016 · 11:150

DOI 10.1007/s11377-016-0055-y

(c) Springer-Verlag Berlin Heidelberg 2016

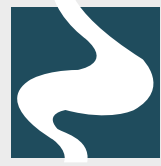

Gastroenterologische

Arbeitsgemeinschaft

Rheinland-Pfalz/Saarland (GARPS)

Kastanienweg 4

67146 Deidesheim

Tel. 06326/962887

www.garps.de

\section{Redaktion:}

Prof. Dr. P. Galle

I. Medizinische Klinik und Poliklinik

Gebäude 301

Langenbeckstr. 1

55131 Mainz
Prof Dr. R. Jakobs

Medizinische Klinik C

Klinikum der Stadt Ludwigshafen

Bremserstraße 79

67063 Ludwigshafen

Prof. Dr. F. Lammert

Klinik für Innere Medizin II

Universitätsklinikum des Saarlandes

Kirrberger Str.

66421 Homburg/Saar

\section{Genetische Faktoren bei komplexen Erkrankungen:}

\section{Molekulare Assoziationsstudie bei Divertikulose und Divertikelkrankheit}

Die Divertikulose und ihre Komplikationen haben als Volkskrankheit eine hohe gesundheitsökonomische Bedeutung mit relevanter Morbidität und Mortalität. Unlängst wurden dazu neue Leitlinien der Deutschen Gesellschaft für Gastroenterologie, Verdauungs- und Stoffwechselkrankheiten (www.dgvs. de/leitlinien) sowie der amerikanischen gastroenterologischen Fachgesellschaft (www.gastro. org/guidelines) veröffentlicht. Die Ursachen der Erkrankung sind erstaunlicherweise immer noch weitgehend ungeklärt. Das einfache Vorhandensein von Divertikeln wird als (asymptomatische) Divertikulose bezeichnet. Der Begriff Divertikelkrankheit fasst die möglichen Komplikationen des Vorhandenseins von Divertikeln, wie die reizdarmähnliche symptomatische Divertikulose, Divertikulitis, Blutung, Perforation, Abszess, Obstruktion und Fistel zusammen. Diese Entitäten sollten strikt getrennt betrachtet werden, da die meisten Divertikelträger lebenslang keine Symptome entwickeln. Auch bezüglich der Therapie der Krankheit sind noch Unsicherheiten verbreitet. Die aktuelle Leitlinienempfehlung, dass eine unkomplizierte
Divertikulitis in manchen Fällen werden kann, scheint noch wenig Anwendung zu finden.

Die Entstehung der Erkrankung wird traditionell überwiegend einer ballaststoffarmen Ernährung zugeschrieben, obgleich die wissenschaftliche Evidenz hierfür gering ist. Genetische Faktoren haben einen hohen Anteil an der Entstehung der Erkrankung, da Unterschiede in der Häufigkeit und Lokalisation der Divertikulose bei verschiedenen ethnischen Gruppen (vorwiegend linksseitig bei Europäern und Amerikanern, überwiegend rechtsseitig bei Asiaten), das gehäufte Auftreten bei angeborenen Bindegewebskrankheiten sowie zahlreiche Fallberichte über das gehäufte Auftreten in Familien vorliegen. Weiter unterstützt wird dies durch Daten aus dem dänischen und schwedischen Zwillingsregister. Zwillingsstudien ermöglichen die Untersuchung multifaktoriell bedingter Erkrankungen und die Abschätzung der Stärke des Einflusses von Umwelt- und genetischen Faktoren. In den Zwillingsstudien zur Divertikulose wurde der Anteil genetischer Faktoren auf $40 \%$ (schwedische Zwillingsstudie) beziehungsweiauch ohne Antibiotika behandelt se $53 \%$ (dänische Zwillingsstudie) berechnet.

Auf molekularer Ebene werden (genomweite) Assoziationsstudien durchgeführt, um genetische Risikofaktoren $\mathrm{zu}$ identifizieren. Bei anderen Volkskrankheiten, wie Diabetes mellitus oder koronarer Herzkrankheit, wurden bereits das Risiko erhöhende Genvarianten identifiziert. Solche Studien wurden jedoch bei der Divertikulose und Divertikelkrankheit bislang noch nicht durchgeführt.

In der Klinik für Innere Medizin II des Universitätsklinikum des Saarlandes werden genetische Ursachen komplexer Erkrankungen untersucht. Wir erforschen im Rahmen einer Assoziationsstudie die genetischen Ursachen der Divertikulose sowie den Zusammenhang mit möglichen Komplikationen der Erkrankung. Dies erfolgt in

\section{Einschlusskriterien für die Assoziationsstudie:}

- Befund einer durchgeführten Koloskopie (Patienten ohne Divertikel können als Kontrollen zur Studie beitragen)

- Die Divertikulose wird anhand der Anzahl der Divertikel in leicht(bis 25 Divertikel), mittel- (25-100 Divertikel) und schwergradig (über 100 Divertikel) eingeteilt.

- Fragebogen

- EDTA-Blut mit Einverständniserklärung zur genetischen Untersuchung (kann per Post eingesandt werden) 